\title{
Enhanced magnifying endoscopy for differential diagnosis of superficial gastric lesions identified with white-light endoscopy
}

\author{
Guo Tao $\cdot$ Lu Xing-hua $\cdot$ Yang Ai-ming $\cdot$ Zhou Wei-xun $\cdot$ Yao Fang $\cdot$ \\ Wu Xi $\cdot$ Wang Li-yin $\cdot$ Lu Chong-mei $\cdot$ Fei Gui-jun $\cdot$ Shu Hui-jun • \\ Wu Dong-sheng $\cdot$ Li Yue $\cdot$ Li Xiao-qing $\cdot$ Qian Jia-ming
}

Received: 6 December 2012/ Accepted: 19 February 2013/Published online: 14 March 2013

(c) The International Gastric Cancer Association and The Japanese Gastric Cancer Association 2013

\begin{abstract}
Background Various techniques using magnifying endoscopy (ME) and chromoendoscopy are being developed to enhance images of gastrointestinal tumor. The aim of this study was to evaluate the diagnostic performance of ME enhanced by acetic acid-indigo carmine mixture (ME-AIM) and ME enhanced with narrow-band imaging (ME-NBI) for differential diagnosis of superficial gastric lesions identified with conventional white-light endoscopy (WLE).

Methods Patients with superficial gastric lesions picked up with WLE were enrolled in the study. ME-NBI and ME-AIM were used to further characterize the lesions. All images of the lesions were evaluated by four skilled endoscopists blinded to the clinical data. The microarchitectural patterns in the lesions were analyzed with reference to the "VS classification" system.

Results A total of 643 lesions (mean diameter, $7 \mathrm{~mm}$ ) from 508 patients (316 men, 192 women; mean age, 63 years) were evaluated. Pathologically, 24 of the 643 lesions were diagnosed as gastric cancer; the others were noncancerous lesions. For diagnosis of gastric cancer, the negative predictive value of each of the three magnified findings (irregular microvascular pattern, irregular
\end{abstract}

G. Tao $\cdot$ L. Xing-hua $(\bowtie) \cdot$ Y. Ai-ming $\cdot$ Y. Fang $\cdot$ W. Xi ·

W. Li-yin · L. Chong-mei · F. Gui-jun · S. Hui-jun ·

W. Dong-sheng · L. Yue · L. Xiao-qing · Q. Jia-ming Department of Gastroenterology, Peking Union Medical College

Hospital, No. 1 Shuaifuyuan, Dong Cheng District,

Beijing 100730, China

e-mail: 1xhbj2000@126.com

Z. Wei-xun

Department of Pathology, Peking Union Medical College

Hospital, Chinese Academy of Medical Sciences,

Beijing 100730, China microsurface pattern, and demarcation line) was high (nearly $100 \%$ ). According to the "VS classification" system, either ME-NBI or ME-AIM had a higher specificity $(99.5 \%$ or $99.4 \%$ vs. $89.5 \%, P<0.001)$ and accuracy $(99.2 \%$ or $98.9 \%$ vs. $89.0 \%, P<0.001)$ than WLE, and ME-AIM was not superior to ME-NBI for identifying carcinoma.

Conclusions Enhanced ME is useful for correctly diagnosing early gastric cancer, and in contrast with ME-AIM, ME-NBI is a more feasible and efficient method for clinical practice.

Keywords Endoscopy $\cdot$ Stomach neoplasms $\cdot$ Diagnosis

$\begin{array}{ll}\text { Abbreviations } \\ \text { AIM } & \text { Acetic acid-indigo carmine mixture } \\ \text { EGC } & \text { Early gastric cancer } \\ \text { IMSP } & \text { Irregular microsurface pattern } \\ \text { IMVP } & \text { Irregular microvascular pattern } \\ \text { ME } & \text { Magnifying endoscopy } \\ \text { NBI } & \text { Narrow-band imaging } \\ \text { NPV } & \text { Negative predictive value } \\ \text { PPV } & \text { Positive predictive value } \\ \text { WLE } & \text { White-light endoscopy }\end{array}$

\section{Introduction}

The detection of early-stage neoplastic lesions in the upper gastrointestinal tract is associated with improved survival and the potential for complete endoscopic resection. Various techniques using magnifying endoscopy (ME) and chromoendoscopy [1-3] are expanding to enhance images of gastric tumors, and observation of the microstructures of gastric mucosa by $\mathrm{ME}[4,5]$ has been proposed in the 
recognition of early gastric cancer (EGC). It has been reported [2] that ME enhanced by acetic acid may be useful for identifying gastric tumors because it shows the superficial mucosal structure more clearly through the acetowhite reaction. A novel chromoendoscopic method using an acetic acid-indigo carmine mixture (AIM) [3] has been reported to improve the diagnostic yield for delineating the margin of EGC. However, the method has not been applied in ME. We therefore speculated that ME enhanced by AIM (ME-AIM) would be an effective method for microsurface architecture analysis and border clarification between the lesion and the surrounding mucosa.

In addition to microsurface architecture, the microvascular structure, observed using magnifying endoscopy enhanced with narrow-band imaging (ME-NBI) [1, 5], has also been reported to be useful for the diagnosis of EGC. NBI is an optical technique that enhances the display of microvascular structures in the superficial mucosal layer through the use of narrow-band filters.

Therefore, the aim of the present study was to evaluate the diagnostic accuracy of enhanced ME (including MEAIM and ME-NBI) in the differential diagnosis of small focal gastric lesions identified with conventional whitelight endoscopy (WLE), according to an assessment of the microsurface pattern and microvascular pattern in the lesions.

\section{Patients and methods}

\section{Patients}

The present study was conducted between March 2010 and June 2012 at Peking Union Medical College Hospital. Patients who received screening endoscopy for EGC using a zoom endoscope were eligible for inclusion. Indications for upper gastrointestinal zoom endoscopy were screening endoscopy for individuals (age $>40$ years) without any obvious symptoms, routine endoscopic examination for patients (age $>40$ years) with anorexia or dyspepsia, and follow-up endoscopy subsequent to a previous pathological diagnosis of intestinal metaplasia. The patients who had received screening endoscopy did not have any history of having being diagnosed as gastric cancer. Referred patients who already were known to have gastric cancer were excluded from this study. Before being enrolled, all patients provided written informed consent.

\section{Endoscopic procedure}

In all patients, screening endoscopy was performed using an upper gastrointestinal zoom endoscope (GIF-H260Z; Olympus, Tokyo, Japan). A soft black hood was mounted on the tip of the scope before the examination, which enabled the endoscopist to fix the focal distance at $3 \mathrm{~mm}$ between the tip of the scope and the gastric mucosal surface. WLE without magnification was performed first in eligible patients. Based on an assessment of the shape (such as flat, depressed, or elevated) and color (pale or reddened), superficial gastric lesions were identified and included for further characterization by experienced endoscopists. When such a lesion was detected during nonmagnifying observation with WLE, mucus adhering to the mucosa was washed away as thoroughly as possible in preparation for the magnifying examination. Conventional ME was carried out first, and the mode was then changed to NBI. For ME-AIM, $20 \mathrm{ml}$ AIM solution (freshly prepared $0.6 \%$ acetic acid added to $0.2 \%$ indigo carmine) [3] was sprinkled evenly over and around the lesion using a washing pipette. The images for the AIM observation were obtained after waiting approximately $2 \mathrm{~min}$ for the color change reaction. All endoscopic images of the whole procedure were recorded in a digital filing system for later evaluation. Biopsy specimens were obtained using standard forceps under magnifying observation from the target lesion where the magnified endoscopic image was obtained.

\section{Analysis of endoscopic images}

Endoscopic diagnosis is open to subjectivity and therefore often shows relatively low interobserver concordance. To avoid possible selection bias and to maintain the quality of the study, the images of each endoscopic modality (including WLE, ME-NBI, ME-AIM), which were arranged randomly on one slide and displayed independently of the images of other endoscopic modality, were evaluated by four skilled endoscopists, who did not have access to the clinical and pathological data. The general consensus of each endoscopic modality was established for an assessment of each lesion as follows.

For the images obtained using WLE, the endoscopic distinctive diagnosis between cancer and non-cancer for each lesion was made based on an assessment of the color and appearance. That is, in comparison with the surrounding mucosa, if at least one of the characteristic endoscopic findings of color change (redness, whiteness, or high color contrast) with uneven surface, irregular margin, or irregular structural pattern was found, we defined the lesions as cancer, and if there were no such findings, we defined the lesions as non-cancer. For the images obtained from ME-NBI and ME-AIM, the microvascular and microsurface pattern of the lesion was respectively evaluated and classified into three types (including regular, irregular, and absent), with reference to the "VS classification" system preferred by Yao et al. [6]. Throughout this article, we consistently describe magnifying endoscopic findings 
according to this system. At the same time, the demarcation line, namely, the border delineation between the lesion and the surrounding mucosa, was identified (present or absent). To evaluate the accuracy of enhanced ME in the differential diagnosis of small focal gastric lesions, we set the criterion (preferred by Yao [6]) for an endoscopic diagnosis of EGC as the presence of an irregular microvascular pattern (IMVP) with a demarcation line and/or the presence of an irregular microsurface pattern (IMSP) with a demarcation line. The study protocol conforms to the ethical guidelines of the 1975 Declaration of Helsinki (6th revision, 2008) and was approved by the institutional review board. Registration was obtained in ClinicalTrials.gov (identifier number: NCT01617876).

\section{Histopathology}

Two forceps biopsy specimens were taken from each lesion and were immediately placed in $10 \%$ buffered formalin. The histopathological diagnosis was made by an experienced gastrointestinal pathologist who was not blinded to clinical information. With reference to the revised Vienna classification [7], lesions diagnosed as low-grade neoplasia (category 3) were excluded from the category of gastric cancer and lesions diagnosed as noninvasive high-grade neoplasia (category 4) were designated as gastric cancer in this study. Pathological diagnosis of biopsy samples were used as the criterion standard for cancer diagnosis.

\section{Statistical analysis}

The significance of differences was tested by using the McNemar test and sign test. $P<0.05$ was considered to indicate significant differences. All statistical analyses were performed using the PASW Statistics 18 software package (IBM, New York, NY, USA). The Wilson score method was used to calculate the $95 \%$ confidence intervals. All authors had access to the study data and reviewed and approved the final manuscript.

\section{Results}

Clinical characteristics of the patients and lesions

In total, 508 patients, from which 643 superficial gastric lesions were detected and evaluated, were enrolled in the study. Of these, 24 lesions were defined pathologically as cancers (category 4), 35 as low-grade neoplasia (category 3), 266 as chronic gastritis with intestinal metaplasia (IM), and 318 as chronic gastritis. The clinicopathological characteristics of the patients and lesions in this study are summarized in Table 1.
Table 1 Clinicopathological characteristics of the patients in the study and lesions

\begin{tabular}{ll}
\hline Patients & 508 \\
Sex (male/female) & $316 / 192$ \\
Mean age (years) & 63 (range, 41-78) \\
Lesions & 643 \\
Mean lesion size (mm) & 7 (range, 3-20) \\
Location & \\
Upper third of the stomach & 84 \\
Middle third of the stomach & 116 \\
Lower third of the stomach & 443 \\
Macroscopic type (Paris classification) & \\
Elevated type (0-IIa) & 303 \\
Flat type (0-IIb) & 148 \\
Depressed type (0-IIc) & 192 \\
Histopathological diagnosis & \\
Chronic gastritis & 318 \\
Chronic gastritis with intestinal metaplasia & 266 \\
Low-grade neoplasia (Category 3) & 35 \\
Gastric cancer (Category 4) & a \\
\hline
\end{tabular}

a According to revised Vienna classification

Microvascular pattern and histopathological findings

For the images obtained using the ME-NBI method, the microvascular pattern of each lesion was evaluated and classified into three types: regular, irregular, or absent, and at the same time the demarcation line of each lesion was identified as present or absent. The relationships between the microvascular patterns and the histopathological findings are shown in Table 2. About $96 \%$ of the nonneoplastic lesions consistently showed a regular microvascular pattern; in contrast, the characteristics of microvascular architecture in $96 \%$ of cancers were irregular or absent. In low-grade neoplasia, only $43 \%$ of lesions showed microvessels that were irregular in either shape or arrangement. All the 24 cancers are delineated by a demarcation line.

Microsurface pattern and histopathological findings

For the images obtained using the ME-AIM method, the microsurface pattern of each lesion was evaluated and classified into one of three types: regular, irregular, or absent, and at the same time the demarcation line of each lesion was identified as present or absent. The relationships between the microsurface patterns and the histopathological findings are shown in Table 3. Similar to the prevalence of microvascular pattern, about $95 \%$ of the nonneoplastic lesions consistently showed a regular microsurface pattern, and $96 \%$ of cancers showed an irregular or absent pattern. In low-grade neoplasia, only $34 \%$ of lesions showed crypt epithelia that were irregular in either shape or 
Table 2 Microvascular patterns and histopathological findings in the lesions

\begin{tabular}{|c|c|c|c|c|}
\hline \multirow[t]{2}{*}{ ME-NBI } & \multicolumn{4}{|c|}{ Histopathology, number (\%) } \\
\hline & $\begin{array}{l}\text { Chronic } \\
\text { gastritis } \\
(n=318)\end{array}$ & $\begin{array}{l}\text { Chronic gastritis } \\
\text { with intestinal } \\
\text { metaplasia } \\
(n=266)\end{array}$ & $\begin{array}{l}\text { Low-grade } \\
\text { neoplasia } \\
(n=35)\end{array}$ & $\begin{array}{l}\text { Gastric } \\
\text { cancer } \\
(n=24)\end{array}$ \\
\hline \multicolumn{5}{|c|}{ Microvascular pattern } \\
\hline Regular & 309 (97.2) & 255 (95.9) & $20(57.1)$ & $1(4.2)$ \\
\hline Irregular & $9(2.8)$ & $11(4.1)$ & $15(42.9)$ & $17(70.8)$ \\
\hline Absent & $0(0)$ & $0(0)$ & $0(0)$ & $6(25.0)$ \\
\hline \multicolumn{5}{|c|}{ Demarcation line } \\
\hline Present & $52(16.4)$ & $108(40.6)$ & $8(22.9)$ & $24(100)$ \\
\hline Absent & $266(83.6)$ & $158(59.4)$ & $27(77.1)$ & $0(0)$ \\
\hline
\end{tabular}

ME-NBI magnifying endoscopy enhanced with narrow-band imaging

Table 3 Microsurface patterns and histopathological findings in the lesions

\begin{tabular}{|c|c|c|c|c|}
\hline \multirow[t]{2}{*}{ ME-AIM } & \multicolumn{4}{|c|}{ Histopathology, number (\%) } \\
\hline & $\begin{array}{l}\text { Chronic } \\
\text { gastritis } \\
(n=318)\end{array}$ & $\begin{array}{l}\text { Chronic gastritis } \\
\text { with intestinal } \\
\text { metaplasia } \\
(n=266)\end{array}$ & $\begin{array}{l}\text { Low-grade } \\
\text { neoplasia } \\
(n=35)\end{array}$ & $\begin{array}{l}\text { Gastric } \\
\text { cancer } \\
(n=24)\end{array}$ \\
\hline \multicolumn{5}{|c|}{ Microsurface pattern } \\
\hline Regular & $311(97.8)$ & $254(95.5)$ & $23(65.7)$ & $1(4.2)$ \\
\hline Irregular & $7(2.2)$ & $12(4.5)$ & $12(34.3)$ & $16(66.7)$ \\
\hline Absent & $0(0)$ & $0(0)$ & $0(0)$ & $7(29.2)$ \\
\hline \multicolumn{5}{|c|}{ Demarcation line } \\
\hline Present & $47(14.8)$ & $104(39.1)$ & $6(17.1)$ & $23(95.8)$ \\
\hline Absent & $271(85.2)$ & $162(60.9)$ & $29(82.9)$ & $1(4.2)$ \\
\hline
\end{tabular}

ME-AIM magnifying endoscopy enhanced by acetic acid-indigo carmine mixture

arrangement. Of 24 cancers, $23(95.8 \%)$ are delineated by a demarcation line.

Diagnostic performance of each of the magnified endoscopic findings for EGC

The diagnostic performance of each of the magnified endoscopic findings for making a diagnosis of EGC is shown in Table 4. Either an irregular microvascular pattern or an irregular microsurface pattern was a remarkable finding for differentiating cancer from noncancerous lesions, which had a high overall accuracy (about $94 \%$ ). The positive predictive value of the three magnified endoscopic findings (IMVP, IMSP, and demarcation line) was not satisfactory, but the negative predictive value of the findings was remarkably high (nearly $100 \%$ ).
Diagnostic accuracy of WLE and enhanced ME for EGC

The accuracy of WLE and enhanced ME (including ME-NBI and ME-AIM) in making a differential diagnosis between cancer and non-cancer for superficial gastric lesions is compared in Table 5. With regard to the diagnosis of early gastric cancer by using either ME-NBI or ME-AIM, the sensitivity analyzed according to both microvascular pattern and microsurface pattern was augmented in contrast with the sensitivity calculated based on microvascular pattern or microsurface pattern alone. On the basis of a VS (vessel plus surface) classification system, the overall accuracy and specificity of either ME-NBI or ME-AIM were significantly higher than that of WLE $(P<0.001)$, as were the sensitivity of both enhanced ME, but the difference had no statistical significance $(P>0.05)$. In the light of outcomes of this study, there is no discrepancy in diagnostic performance between ME-NBI and ME-AIM.

\section{Discussion}

When performing screening endoscopy, small focal gastric lesions are frequently encountered. To diagnose EGC, numerous biopsies are taken from such lesions, which mean that a correct diagnosis is usually made based on histopathological, and not endoscopic, findings. Previous studies [2, 4, 5] have shown that observation of the microstructures of gastric mucosa by $\mathrm{ME}$ is useful in the recognition of EGC, which is based on two distinctly different morphological characteristics, that is, mucosal microsurface structural change and microvascular structural change. The loss of a clear regular microstructural pattern is characteristic of EGC, and a systematic but simple classification system (the VS classification system), as well as a diagnostic criterion based on microvascular pattern and microsurface pattern, has been proposed by Yao et al. [6]. Therefore, we investigated the diagnostic accuracy of magnified endoscopic findings for differentiating between cancer and noncancerous lesions according to this system.

In our experience, the fine microstructural pattern is often too subtle to be detected clearly with a standard magnifying endoscope. Enhanced ME that yields clearer images of microstructures on a mucosal surface has been shown to be capable of predicting the histological characteristics of EGC and recognizing the demarcations easily $[1,2,8]$. It has been reported [2, 9] that acetic acid spray appears to improve surface pattern visualization by means of the aceto-white reaction. Noncancerous gastric mucosa is believed to whiten when it comes into contact with acetic acid. However, cancer cells do not, producing a good 
Table 4 Diagnostic performance of each of the magnified endoscopic findings for early gastric cancer

\begin{tabular}{|c|c|c|c|c|c|}
\hline Method & Sensitivity $(95 \% \mathrm{CI})$ & Specificity $(95 \%$ CI $)$ & $\begin{array}{l}\text { PPV } \\
(95 \% \text { CI })\end{array}$ & $\begin{array}{l}\text { NPV } \\
(95 \% \text { CI })\end{array}$ & Accuracy $(95 \% \mathrm{CI})$ \\
\hline \multicolumn{6}{|l|}{ ME-NBI } \\
\hline $\mathrm{IMVP}^{\mathrm{a}}$ & $\begin{array}{l}70.8 \% \\
(50.8-85.1)\end{array}$ & $\begin{array}{l}94.4 \% \\
(92.2-95.9)\end{array}$ & $\begin{array}{l}32.7 \% \\
(92.2-95.9)\end{array}$ & $\begin{array}{l}98.8 \% \\
(97.6-99.4)\end{array}$ & $\begin{array}{l}93.5 \% \\
(91.3-95.1)\end{array}$ \\
\hline Demarcation line ${ }^{b}$ & $\begin{array}{l}100 \% \\
(86.2-100)\end{array}$ & $\begin{array}{l}72.9 \% \\
(69.2-76.2)\end{array}$ & $\begin{array}{l}12.5 \% \\
(8.5-17.9)\end{array}$ & $\begin{array}{l}100 \% \\
(99.2-100)\end{array}$ & $\begin{array}{l}73.9 \% \\
(70.3-77.1)\end{array}$ \\
\hline \multicolumn{6}{|l|}{ ME-AIM } \\
\hline $\mathrm{IMSP}^{\mathrm{a}}$ & $\begin{array}{l}66.7 \% \\
(46.7-82.0)\end{array}$ & $\begin{array}{l}95.0 \% \\
(93.0-96.5)\end{array}$ & $\begin{array}{l}34.0 \% \\
(22.2-48.3)\end{array}$ & $\begin{array}{l}98.7 \% \\
(97.4-99.3)\end{array}$ & $\begin{array}{l}93.9 \% \\
(91.8-95.5)\end{array}$ \\
\hline Demarcation line ${ }^{\mathrm{b}}$ & $\begin{array}{l}95.8 \% \\
(79.8-99.3)\end{array}$ & $\begin{array}{l}74.6 \% \\
(71.1-77.9)\end{array}$ & $\begin{array}{l}12.8 \% \\
(8.7-18.4)\end{array}$ & $\begin{array}{l}99.8 \% \\
(98.8-100)\end{array}$ & $\begin{array}{l}75.4 \% \\
(72.0-78.6)\end{array}$ \\
\hline
\end{tabular}

$M E$-NBI magnifying endoscopy enhanced with narrow-band imaging, ME-AIM magnifying endoscopy enhanced by acetic acid-indigo carmine mixture, $I M S P$ irregular microsurface pattern, $I M V P$ irregular microvascular pattern, $P P V$ positive predictive value, $N P V$ negative predictive value

${ }^{a}$ Positive was defined as irregular, and negative was defined as either regular or absent

${ }^{\mathrm{b}}$ Positive was defined as present, and negative was defined as absent

Table 5 Diagnostic accuracy of white-light endoscopy (WLE) and enhanced magnifying endoscopy (ME) for early gastric cancer

\begin{tabular}{|c|c|c|c|c|c|}
\hline Method & $\begin{array}{l}\text { Sensitivity (95\% } \\
\text { CI) }\end{array}$ & $\begin{array}{l}\text { Specificity }(95 \% \\
\text { CI) }\end{array}$ & $\begin{array}{l}\text { PPV } \\
(95 \% \mathrm{CI})\end{array}$ & $\begin{array}{l}\text { NPV } \\
(95 \% \mathrm{CI})\end{array}$ & $\begin{array}{l}\text { Accuracy (95\% } \\
\text { CI) }\end{array}$ \\
\hline WLE & $\begin{array}{l}75.0 \% \\
(55.1-88.0) \\
(18 / 24)\end{array}$ & $\begin{array}{l}89.5 \% \\
(86.8-91.7) \\
(554 / 619)\end{array}$ & $\begin{array}{l}21.7 \% \\
(14.2-31.7) \\
(18 / 83)\end{array}$ & $\begin{array}{l}98.9 \% \\
(97.7-99.5) \\
(554 / 560)\end{array}$ & $\begin{array}{l}89.0 \% \\
(86.3-91.2) \\
(572 / 643)\end{array}$ \\
\hline \multicolumn{6}{|l|}{ ME-NBI } \\
\hline $\mathrm{IMVP}+\mathrm{DL}$ & $\begin{array}{l}70.8 \% \\
(50.8-85.1) \\
(17 / 24)\end{array}$ & $\begin{array}{l}99.7 \% \\
(98.8-99.9) \\
(617 / 619)\end{array}$ & $\begin{array}{l}89.5 \% \\
(68.6-97.1) \\
(17 / 19)\end{array}$ & $\begin{array}{l}98.9 \% \\
(97.7-99.5) \\
(617 / 624)\end{array}$ & $\begin{array}{l}98.6 \% \\
(97.4-99.3) \\
(634 / 643)\end{array}$ \\
\hline $\mathrm{IMVP}+\mathrm{DL}$ or IMSP $+\mathrm{DL}$ & $\begin{array}{l}91.7 \% \text { b,c } \\
(74.2-97.7) \\
(22 / 24)\end{array}$ & $\begin{array}{l}99.5 \%^{\mathrm{a}} \\
(98.6-99.8) \\
(616 / 619)\end{array}$ & $\begin{array}{l}88.0 \% \\
(70.0-95.8) \\
(22 / 25)\end{array}$ & $\begin{array}{l}99.7 \% \\
(98.8-99.9) \\
(616 / 618)\end{array}$ & $\begin{array}{l}99.2 \%^{\mathrm{a}} \\
(98.2-99.7) \\
(638 / 643)\end{array}$ \\
\hline \multicolumn{6}{|l|}{ ME-AIM } \\
\hline $\mathrm{IMSP}+\mathrm{DL}$ & $\begin{array}{l}66.7 \% \\
(46.7-82.0) \\
(16 / 24)\end{array}$ & $\begin{array}{l}99.5 \% \\
(98.6-99.8) \\
(616 / 619)\end{array}$ & $\begin{array}{l}84.2 \% \\
(62.4-94.5) \\
(16 / 19)\end{array}$ & $\begin{array}{l}98.7 \% \\
(97.5-99.4) \\
(616 / 624)\end{array}$ & $\begin{array}{l}98.3 \% \\
(97.0-99.0) \\
(632 / 643)\end{array}$ \\
\hline $\mathrm{IMVP}+\mathrm{DL}$ or IMSP $+\mathrm{DL}$ & $\begin{array}{l}87.5 \%^{\mathrm{b}, \mathrm{d}} \\
(69.0-95.7) \\
(21 / 24)\end{array}$ & $\begin{array}{l}99.4 \%^{\mathrm{a}} \\
(98.4-99.8) \\
(615 / 619)\end{array}$ & $\begin{array}{l}84.0 \% \\
(65.4-93.6) \\
(21 / 25)\end{array}$ & $\begin{array}{l}99.5 \% \\
(98.6-99.8) \\
(615 / 618)\end{array}$ & $\begin{array}{l}98.9 \%^{\mathrm{a}} \\
(97.8-99.5) \\
(636 / 643)\end{array}$ \\
\hline \multicolumn{6}{|l|}{ ME-NBI plus ME-AIM } \\
\hline $\begin{array}{l}\text { IMVP + DL (ME-NBI) or IMSP + DL (ME- } \\
\text { AIM) }\end{array}$ & $\begin{array}{l}87.5 \% \\
(69.0-95.7) \\
(21 / 24)\end{array}$ & $\begin{array}{l}99.4 \% \\
(98.4-99.8) \\
(615 / 619)\end{array}$ & $\begin{array}{l}84.0 \% \\
(65.4-93.6) \\
(21 / 25)\end{array}$ & $\begin{array}{l}99.5 \% \\
(98.6-99.8) \\
(615 / 618)\end{array}$ & $\begin{array}{l}98.9 \% \\
(97.8-99.5) \\
(636 / 643)\end{array}$ \\
\hline
\end{tabular}

$W L E$ white-light endoscopy, $M E$-AIM magnifying endoscopy enhanced by acetic acid-indigo carmine mixture, $M E-N B I$ magnifying endoscopy enhanced with narrow-band imaging, IMSP irregular microsurface pattern, $I M V P$ irregular microvascular pattern, $D L$ demarcation line, $P P V$ positive predictive value, $N P V$ negative predictive value

${ }^{a} P<0.001$ vs. WLE

${ }^{\mathrm{b}} P>0.05$ vs. WLE

${ }^{\mathrm{c}} P>0.05$ vs. IMVP $+\mathrm{DL}$

${ }^{\mathrm{d}} P>0.05$ vs. IMSP $+\mathrm{DL}$ 
contrast between a cancer lesion and the surrounding noncancerous mucosa. Indigo carmine, which does not stain the mucosa but collects in mucosal grooves, makes this contrast even clearer. Furthermore, ME is particularly useful to accentuate the microstructure and recognize this contrast. Thus, we speculated that the combination of indigo carmine and acetic acid application for mucosal surface pattern analysis would be an efficient diagnostic method and that it would be possible to demonstrate the microsurface pattern clearly and determine the lesion border easily by using the ME-AIM method. As ME-NBI gives better visualization of blood vessels as a result of the high absorption of blue and green light by hemoglobin, it was used to assess the microvascular pattern in this study.

In the present study, almost all the nonneoplastic lesions, including chronic gastritis and chronic gastritis with IM, consistently showed both regular microvascular pattern and regular microsurface pattern (Figs. 1, 2). Although $16 \%(52 / 318)$ of chronic gastritis and $41 \%$
(108/266) of chronic gastritis with IM presented a clear demarcation line, neither an IMVP nor an IMSP could be seen in both lesions and the surrounding mucosa (Fig. 2). Of the 35 low-grade neoplasias, $43 \%(15 / 35)$ had an irregular microvascular pattern and $34 \%(12 / 35)$ had an irregular microsurface pattern (Fig. 3). However, only $23 \%(8 / 35)$ of these lesions demonstrated a demarcation line. In $92 \%$ (22/24) of gastric cancers, either an irregular microvascular pattern with a demarcation line or an irregular microsurface pattern with a demarcation line could be identified (Fig. 4), which met the criterion for EGC according to the "VS classification" system. Our study showed that the presence of an IMVP or an IMSP alone was an important magnifying endoscopic finding for differentiating between gastritis and EGC, but it was still difficult to distinguish EGC from low-grade dysplasia, which could account for the low PPV of the IMVP or IMSP for diagnosing EGC. While combining the presence of a microstructural pattern with the presence of a demarcation
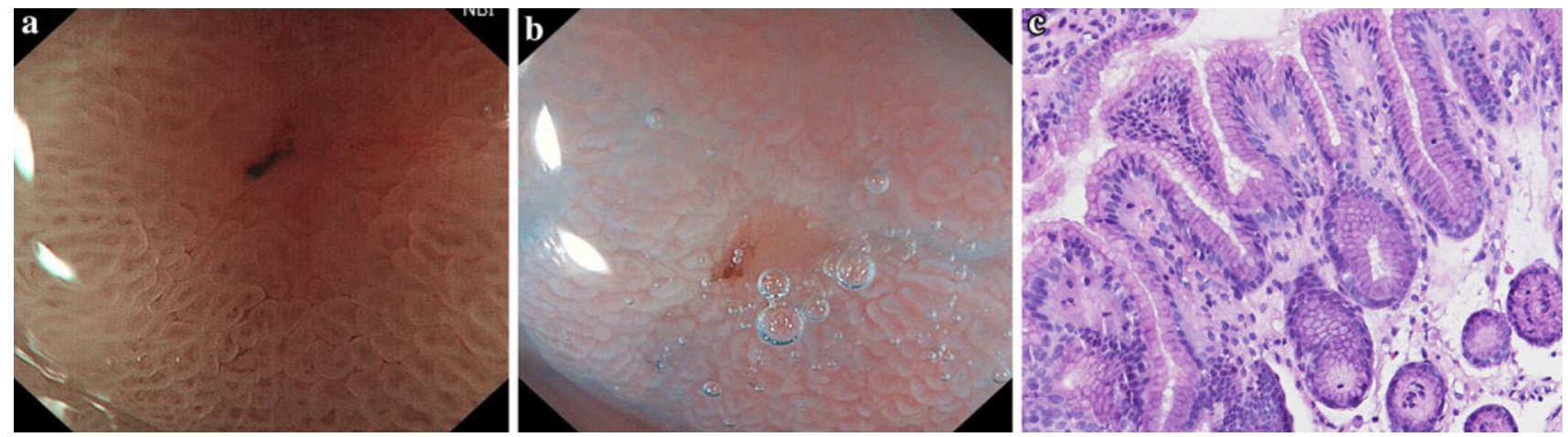

Fig. 1 Endoscopic and histopathological findings of a focal lesion (type 0-IIa, $6 \mathrm{~mm}$ in diameter) within the gastric antrum. a Magnifying endoscopy enhanced with narrow-band imaging (NBI): regular microvascular pattern plus regular microsurface pattern, without a demarcation line. b Magnifying endoscopy enhanced with acetic acid-indigo carmine mixture (AIM): regular microsurface pattern without a demarcation line. c Histopathological diagnosis of the biopsy specimen was chronic gastritis (revised Vienna classification, C1)
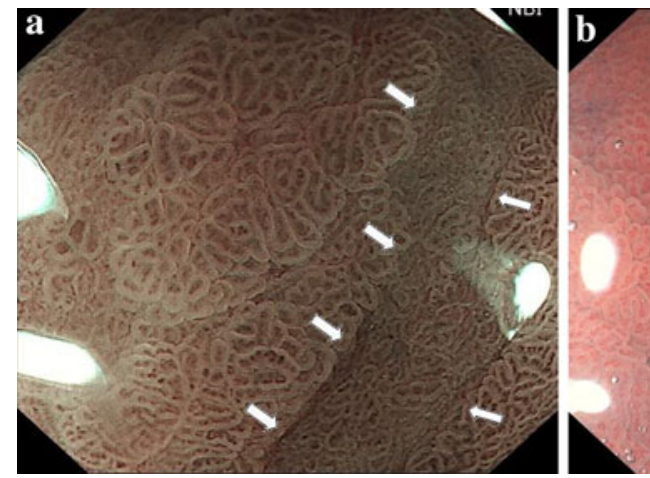

Fig. 2 Endoscopic and histopathological findings of a focal lesion (type 0-IIc, $8 \mathrm{~mm}$ in diameter) within the gastric body. a Magnifying endoscopy enhanced with NBI: regular microvascular pattern plus regular microsurface pattern, with a demarcation line (arrows). b Magnifying endoscopy enhanced with AIM: regular microsurface pattern with a demarcation line (arrows). c Histopathological diagnosis of the biopsy specimen was chronic gastritis with intestinal metaplasia (revised Vienna classification, C1) 

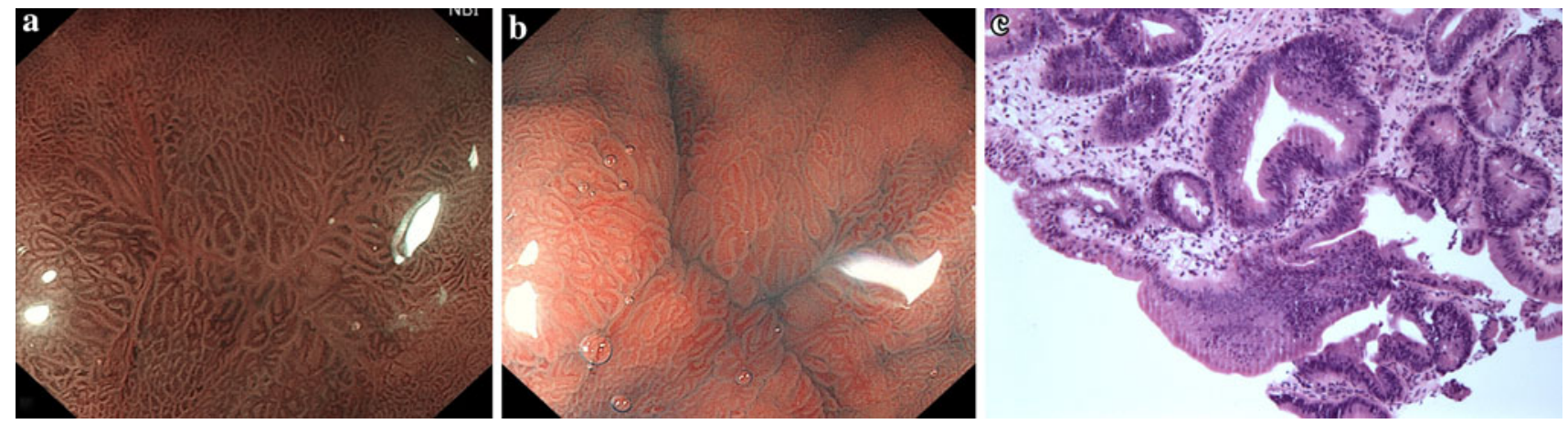

Fig. 3 Endoscopic and histopathological findings of a focal lesion (type 0-IIb, $6 \mathrm{~mm}$ in diameter) within the gastric antrum. a Magnifying endoscopy enhanced with NBI: regular microvascular pattern plus irregular microsurface pattern, without a demarcation line. b Magnifying endoscopy enhanced with AIM: irregular microsurface pattern without a demarcation line. c Histopathological diagnosis of the biopsy specimen was low-grade neoplasia (revised Vienna classification, C3)
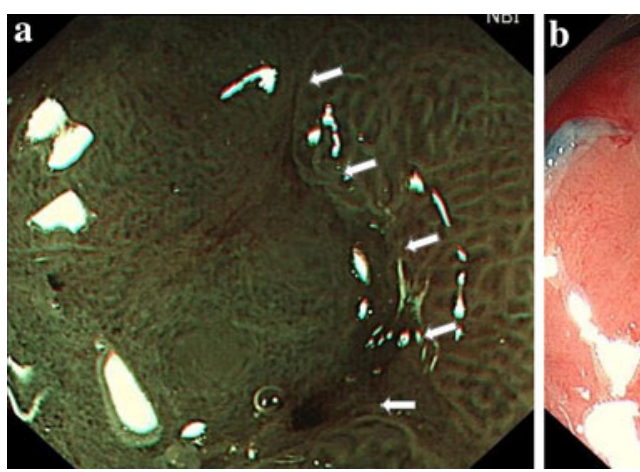

Fig. 4 Endoscopic and histopathological findings of a focal lesion (type 0-IIc, $12 \mathrm{~mm}$ in diameter) within the gastric antrum. a Magnifying endoscopy enhanced with NBI: irregular microvascular pattern plus irregular microsurface pattern, with a demarcation line (arrows).

line as a diagnostic criterion, the differential diagnostic performance between EGC and low-grade neoplasia could be improved.

For the diagnosis of EGC, the negative predictive value for the presence of each of the three magnified endoscopic findings (IMVP, IMSP, and demarcation line) was high enough (nearly $100 \%$ ) to suggest that when we fail to identify an IMVP, an IMSP, or a demarcation line in a superficial gastric lesion, then the lesion is not cancer. Furthermore, we do not need to take a biopsy specimen from a lesion that shows a regular microstructural pattern in the absence of a demarcation line between the lesion and the surrounding mucosa. Although the microvascular pattern or microsurface pattern alone is useful for diagnosing EGC by enhanced ME, the sensitivity is relatively low because we sometimes encounter difficulties in visualizing the microstructure of the lesion, namely, the absence of a microarchitectural pattern. In such cases, we should analyze both the microvascular pattern and microsurface pattern together. For instance, if the microvascular pattern is not visualized, the microsurface pattern can be a good b Magnifying endoscopy enhanced with AIM: irregular microsurface pattern with a demarcation line (arrows). c Histopathological diagnosis of the biopsy specimen was high-grade neoplasia (revised Vienna classification, C4)

alternative. This finding explains why the sensitivity of ME-NBI or ME-AIM was augmented by employing the comprehensive VS classification system involving both the microvascular and microsurface pattern.

The diagnostic accuracy of WLE for EGC (75 \% sensitivity and $90 \%$ specificity) in the present study was higher than that in another study [10]. The reason may be attributed to differences in study design and inclusion criteria. For example, our study included flat, depressed, and elevated lesions, but the other study included mainly flat or depressed lesions. As we know empirically, the differential diagnosis of EGC is especially difficult in the case of superficial depressed lesions, so the diagnostic accuracy in the other study could be lower. In contrast to the diagnostic performance of WLE, VS (vessel plus surface) classification system-based diagnosis by enhanced ME (either MENBI or ME-AIM) achieved superior overall accuracy $(99 \%, p<0.001)$ and specificity $(99 \%, p<0.001)$. The sensitivity $(92 \%, P>0.05)$ was also superior, but the difference had no statistical significance because of the relatively small case numbers of EGC. The advantage of 
ME-NBI over WLE for diagnosing EGC has been demonstrated previously. This study is the first evaluation of the diagnostic performance between the two enhanced ME methods, ME-NBI and ME-AIM. Based on our results, ME-AIM was not superior to ME-NBI for identifying EGC. Because ME-AIM has the disadvantage of dye spraying and is time consuming, and ME-NBI can simply visualize the microsurface pattern as well as the microvascular pattern, we think ME-NBI is a more feasible and efficient method for clinical practice.

The high diagnostic accuracy of enhanced ME leads us to believe that we may even be able to perform an endoscopic resection for small lesions when the lesions do actually fulfill the diagnostic criteria for EGC. It has been reported [4] that in some cases the therapeutic decision with regard to endoscopic resection was made based not on the histopathological findings but on magnifying endoscopic diagnosis alone. A simple explanation could be insufficient sampling through small biopsy samples compared with endoscopic resection specimens. Although the use of enhanced ME has been applied in the diagnosis of EGC, these technologies for diagnostic purposes are not yet fully utilized because of the requirement of special equipment and technical difficulties when manipulating magnifying endoscopes. Therefore, WLE is still the first choice for EGC screening because of its simplicity and high negative predictive value, and biopsy should be compulsory for any lesion suspected of being cancerous if magnifying endoscopy is not at hand. However, when endoscopic resection is considered to be a candidate therapy, enhanced ME is a useful method for characterization, confirmation, and delineation of the lesion.

We must consider some limitations of this study carefully. First, the study is not a real-time diagnostic yield but a retrospective evaluation using recorded images. Second, the case number of EGC is small and the data are from a single center. Therefore, further multicenter studies with a large number of cases are needed to fortify the evidence. Third, "irregularity" of a microvascular or microsurface pattern, the key word in the criterion, is a conceptual term, and the criterion may make diagnosis subjective.

In conclusion, enhanced ME (either ME-NBI or ME-AIM) is useful for correctly distinguishing, on the basis of "VS classification" system, between EGC and non-carcinomatous lesions when a superficial gastric lesion is seen at routine screening with conventional WLE. By comparison to ME-AIM, ME-NBI is a more feasible and efficient method for clinical practice.

Acknowledgments This work was supported by the professionspecific funded projects in standardization of targeted therapy and cell therapy and applied research of early diagnosis and treatment for cancer from Chinese Ministry of Health (Grant 200902002).

\section{References}

1. Nakayoshi T, Tajiri H, Matsuda K, Kaise M, Ikegami M, Sasaki $\mathrm{H}$, et al. Magnifying endoscopy combined with narrow band imaging system for early gastric cancer: correlation of vascular pattern with histopathology (including video). Endoscopy. 2004;36:1080-4.

2. Tanaka K, Toyoda H, Kadowaki S, Kosaka R, Shiraishi T, Imoto I, et al. Features of early gastric cancer and gastric adenoma by enhanced-magnification endoscopy. J Gastroenterol. 2006;41: $332-8$.

3. Kawahara Y, Takenaka R, Okada H, Kawano S, Inoue M, Tsuzuki T, et al. Novel chromoendoscopic method using an acetic acid-indigo carmine mixture for diagnostic accuracy in delineating the margin of early gastric cancers. Dig Endosc. 2009; 21:14-9.

4. Yao K, Iwashita A, Tanabe H, Nagahama T, Matsui T, Ueki T, et al. Novel zoom endoscopy technique for diagnosis of small flat gastric cancer: a prospective, blind study. Clin Gastroenterol Hepatol. 2007;5:869-78.

5. Kaise M, Kato M, Urashima M, Arai Y, Kaneyama H, Kanzazawa $Y$, et al. Magnifying endoscopy combined with narrow-band imaging for differential diagnosis of superficial depressed gastric lesions. Endoscopy. 2009;41:310-5.

6. Yao K, Anagnostopoulos GK, Ragunath K. Magnifying endoscopy for diagnosing and delineating early gastric cancer. Endoscopy. 2009;41:462-7.

7. Dixon MF. Gastrointestinal epithelial neoplasia: Vienna revisited. Gut. 2002;51:130-1.

8. Kadowaki S, Tanaka K, Toyoda H, Kosaka R, Imoto I, Hamada $\mathrm{Y}$, et al. Ease of early gastric cancer demarcation recognition: a comparison of four magnifying endoscopy methods. J Gastroenterol Hepatol. 2009;24:1625-30.

9. Yagi K, Aruga Y, Nakamura A, Sekine A, Umezu H. The study of dynamic chemical magnifying endoscopy in gastric neoplasia. Gastrointest Endosc. 2005;62:963-9.

10. Kato M, Kaise M, Yonezawa J, Toyoizumi H, Yoshimura N, Yoshida $\mathrm{Y}$, et al. Magnifying endoscopy with narrow-band imaging achieves superior accuracy in the differential diagnosis of superficial gastric lesions identified with white-light endoscopy: a prospective study. Gastrointest Endosc. 2010;72:523-9. 